\title{
An Efficient FD-aided Visible Light Communication System
}

\author{
Xu Wanga , Yang Li ${ }^{\mathrm{a}}$, Min Feng ${ }^{\mathrm{b}}$, Jianye Zhang ${ }^{\mathrm{c}}$, and Wang Luo ${ }^{\mathrm{b}}$ ** \\ ${ }^{a}$ National State Grid Xinjiang Electric Power Research Institute, Urumchi, 830011, China \\ ${ }^{b}$ NARI Group Corporations/State Grid Electric Power Research Institute, Nanjing, 210003, China \\ ${ }^{c}$ National State Grid Xinjiang Electric Power Co. Ltd., Urumchi, 830063, China
}

\begin{abstract}
The sharply increasing implementation of light emitting diodes (LED) boosts the rapid development of the visible light communication (VLC). However, current VLC systems fail to achieve high data rate and large capacity region, which are prohibited by the fact that the dynamically changing VLC wireless channel and the statistics of the visible lights. For the sake of breaking these fundamental limitations, in this paper, we develop a novel VLC system for achieving high-speed and reliable communication. To be specific, taking the advantages of the full-duplex (FD) communication that can significantly enhance the spectrum efficiency, we exploit a novel FD-aided VLC system. Also, the developed VLC system is capable of realizing transmitting network signals directly. Additionally, we test the proposed VLC system in practice, and experiments results demonstrate that the developed VLC system outperforms other strategies in terms of the data rate and reliability.
\end{abstract}

Keywords: Visible Light Communication (VLC); Full-Duplex (FD) communications; data rate

(Submitted on March 7, 2018; Revised on April 21, 2018; Accepted on May 23, 2018)

(C) 2018 Totem Publisher, Inc. All rights reserved.

\section{Introduction}

In recent decades, optical wireless communication has been regarded as an emerging technology motivated by the advance in the fabrication and development of solid-state lighting devices, i.e., light emitting diode (LED), laser diode (LD), and photodetector (PD) [8]. In particular, VLC has been considered a candidate for future wireless access [6], which is dedicated by its dual functionality, and the high-rate data transmission. Furthermore, LED has high sensitivity, good modulation performance and high modulation bandwidth [3], which has been witnessed as a very promising technique for the VLC.

Inspired by the super performance of the VLC, large amount of researches have been devoted to investigating its performance. In [5], the authors proposed a novel scheme to realize data transmission and environment perception simultaneously. For the sake of resource allocation issue, a heterogeneous software-defined and twinned VLC and RF small-cell networks has been provided [13]. Also, taking the advantages of Non-orthogonal multiple access (NOMA), paper [4] presented a VLC-NOMA system for achieving better SER performance. Then, since the adoption of orthogonal frequency division multiplexing (OFDM) and relaying technique can greatly enhance system performance, the authors proposed a relay-assisted VLC system based on asymmetrically clipped direct current biased optical OFDM for 5G wireless networks [7]. Furthermore, in [11], a Fast Optical Orthogonal Frequency Division Multiplexing (FOOFDM) was developed and the authors investigated Probability of Error expression with various channel estimation.

Among these appealing techniques, high-speed and reliable VLC systems aroused great interests among the academia and industries. In [2], the authors employed the orthogonal circulated matrix transform (OCT) precoding to enhance the data rate of the VLC system. Meanwhile, a novel EGC-STBC for a $2 \times 2$ MIMO VLC network was proposed and it can achieve $1.8 \mathrm{Gbit} / \mathrm{s}$ data rate. Moreover, in [9], the authors developed multi-user access based on multi-band CAP modulation over a wavelength division multiplexing (WDM) VLC system and its data rate is up to $1.35 \mathrm{Gbit} / \mathrm{s}$. In addition, by deploying an LED array transmitter and a high-speed image sensor receiver, paper [12] derived a strategy for avoiding vehicle vibration

\footnotetext{
* Corresponding author.

E-mail address: luowang@sgepri.sgcc.com.cn
} 
from road irregularity and achieving high-speed communication.

Motivated by the above considerations, in this paper, we develop an efficient VLC system that integrates the fullduplex (FD) communication into VLC systems for realizing high-speed and reliable transmission under various kinds of signals. Briefly speaking, the contribution of this paper can be listed as follows.

- By using two symmetric equipment, we design a novel VLC system, which enables FD communication. Also, experiments results corroborate its super performance in terms of efficiency and data rate.

- Different from previous works, this novel VLC system is capable of dealing with network signals directly. Hence, it can be used as an access node for accessing the Internet without requiring optical fibers.

The rest of this paper is organized as follows. In Section 2, we propose a FD-aided VLC system, in which two symmetric VLC systems are equipped. Then, in Section 3, we describe several important schemes for the corresponding blocks of the VLC system. Experiments results for evaluating the performance of the proposed schemes are provided in Section 4, which is followed by conclusions in Sections 5.

\section{System Model}

As shown in Figure. 1, we consider a VLC system, which comprises a network access module, a network signal processing module, a transmitter, two LED, two photodiodes (PDs), a receiver, an electronic signal processing module, a local processing and display module, as well as a cloud server module. To be specific, the transmitted signals can arrive at the receiver via the visible light channel.

Here, there are two LED and two PDs being implemented at this VLC system. Consider the forward link. It includes one LED at the transmitter and one PD at the receiver, while the reverse link is constituted by the LED at the receiver and the PD at the transmitter to realize communication interaction. For the sake of showing its working procedures, its procedures can be presented as follows.

Step1 The Internet signals are conveyed into the network access module.

Step2 The processed signals are converted into digital signals with the aids of the network signal processing module.

Step3 The digital signals are turned into optical signals at the signal receivers. Also, the LED emit the light intensity signals.

Step4 The light intensity signals corrupted by additive white Gaussian noise (AWGN) are received at the PD of the system.

Step5 The received signals at the PD are added into the cloud server module to upload the processed data such as the bit error ratio (BER), and the latency.

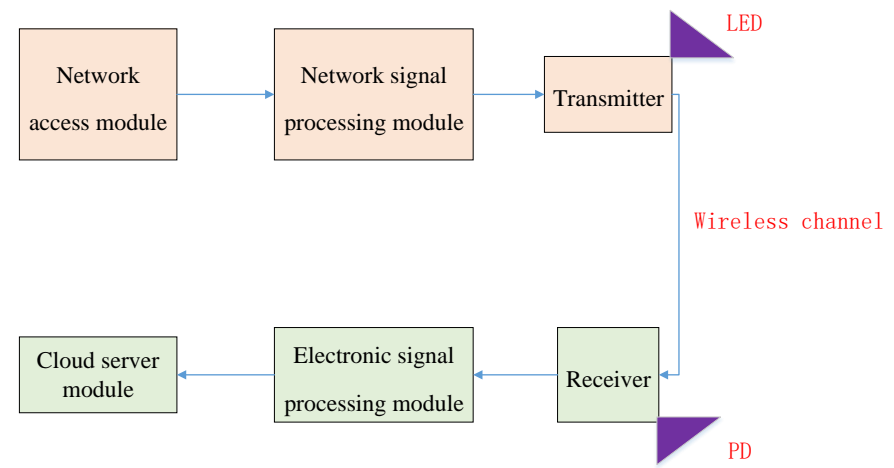

Figure 1. The developed VLC framework

It is noted that single side of the proposed VLC system is designed as the half-duplex manner, which means that only deploying two symmetric equipment can we realize FD communication. This mechanism contributes to the higher spectrum and energy efficiency of the communication system. As depicted in Figure. 2, this is the 3-dimension (3D) model of the developed VLC system. Thus, the VLC system can carry out FD communication. 


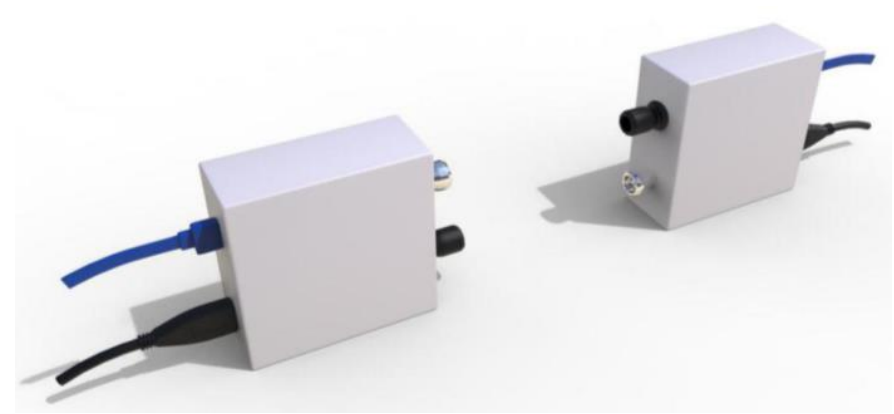

Figure 2. The proposed VLC system

\section{Implementation of the VLC System}

In this section, for the sake of realizing efficient communication performance, we design a novel VLC system and its technical methods are as follows.

\subsection{Power Supply Scheme}

As shown in Figure. 3, we use USB-micro for power supply at the transmitter and the receiver, at which the $3.3 \mathrm{~V}$ and $5 \mathrm{~V}$ sources are employed for the Li+ battery. To be specific, the ICI chip MAX1811 is deployed for power supplying for the Li+ battery. When we use USB for power supplying for the battery, the low-power SETI side of the ICI chip should be lowered, and rated current is set as $100 \mathrm{~mA}$ while it would be $500 \mathrm{~mA}$ at the high-power SETI side. Similar to this principle, we adapt the level of the SELV side as $4.2 \mathrm{~V}$ and $4.1 \mathrm{~V}$, respectively, and the precision of the IC1 to $0.5 \%$. When charging, the LED are lighten.

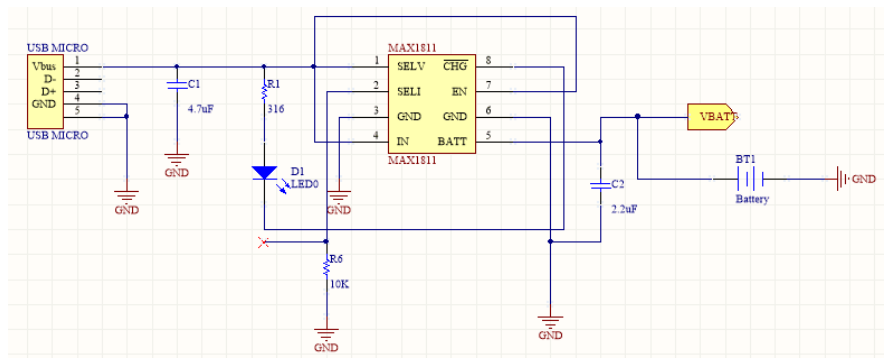

Figure 3. MAX811USB charging IC circuit

Since the voltage of the Li+ battery is $3.7 \mathrm{~V}$, the standard voltage are $5 \mathrm{~V}$ and $3.7 \mathrm{~V}$, respectively. Thus, we adopt the IC2 DC-DC converter to enhance the level of the power of the battery. It can improve the voltage $V_{\text {batt }}$ of the battery to $5 \mathrm{~V}$, and $450 \mathrm{~mA}$ current is output as $V_{\text {out }}$.

The low-power battery detection circuit and switch would protect the Li+ battery from strongly discharging, which can be realized by disconnecting the battery. The low battery threshold (LB1 pin level) is determined by the external resistance R3 and R4 between the $V_{\text {batt }}$ and the GND. By connecting the output pin LBO to the switch pin SHDN, we can break off the IC2 and the load.

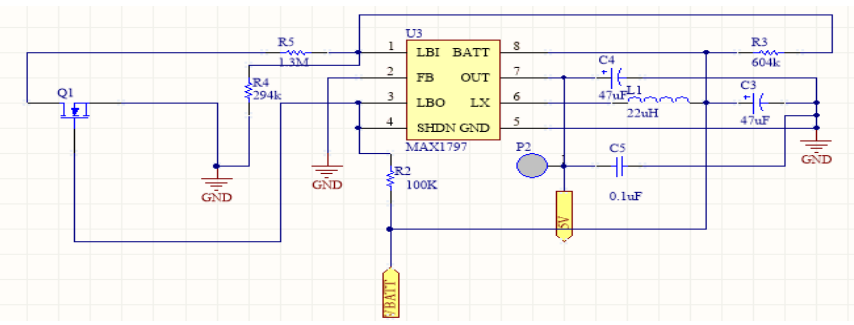

Figure 4. Li+ battery boost circuit 
When the low-power battery and load are disconnected by the low-power battery detection circuit, vibration would be occurred at the IC2 induced by the resistance of the Li+ battery. This is because that the voltage of the battery would improve when the voltage induced by the battery resistance disappears. Hence, the vibration would be removed at the Nchannel MOSFET by introducing feedback at the detection circuit. It can be observed from Figure. 4 that the threshold voltage is set as $2.9 \mathrm{~V}$. When the voltage $V_{\text {batt }}$ reduces to less than $2.9 \mathrm{~V}$, the LBO would open to enhance the SHDN for breakovering the MOSFET. When the MOSFET breakovers, the parallel circuit is formed by the R5(1.3M $\Omega)$ and $(249 \mathrm{k} \Omega)$. In this way, the voltage of the threshold of the pin LB1 would be improved to $3.3 \mathrm{~V}$ and the vibration would be removed.

Then, as exhibited in Figure. 5, we can see that the DC-DC buck converter is the chip MAX1837, which can reduce the voltage from $5 \mathrm{~V}$ to $3.3 \mathrm{~V}$ and output $250 \mathrm{~mA}$ circuit to the load, achieving over $90 \%$ in terms of energy efficiency.

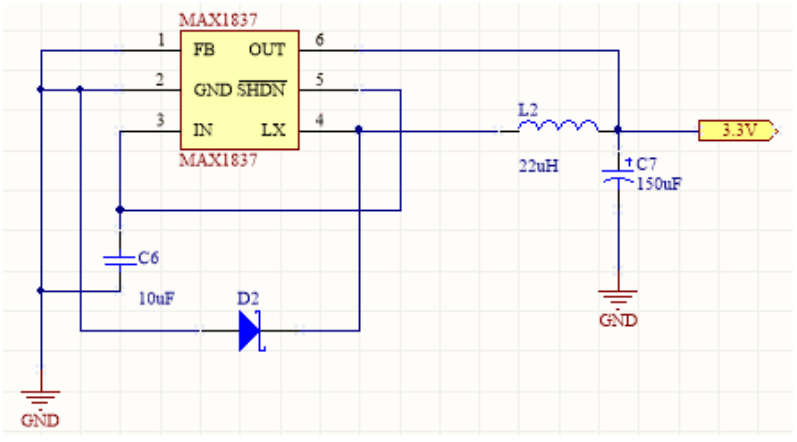

Figure 5. Buck circuit from $5 \mathrm{~V}$ to $3.3 \mathrm{~V}$

\subsection{Carriers Signals Generating Circuit}

This subsection presents the framework of an equipment for providing carriers to the VLC system, as shown in Figure. 6 . When the voltage is $3.3 \mathrm{~V}$, the high level of the circuit is higher than $2.4 \mathrm{~V}$ while the low level is below to $0.4 \mathrm{~V}$. It can provide stable $100 \mathrm{MHz}$ rectangular carriers and input these carriers to the XPLS and XMNS pins of the analog broadband multiplex for modulation.

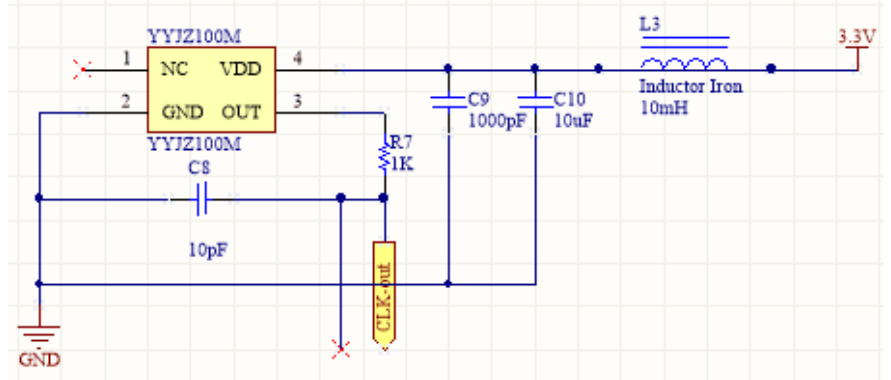

Figure 6. Carriers signals generating circuit

\subsection{Broadband Analog Multiplexing Circuit Based on ADL5391}

For the sake of improving frequency response of the LED, we should set the forward bias voltage and then mix the modulated TTL signals. Thus, according to the main characteristics of the ADL5391, we adopt the strategy as follows.

To begin with, we use the $5 \mathrm{~V}$ single source for power supply at the multiplex chip, and the transfer function is expressed as

$$
V_{\mathrm{W}}=a\left(V_{X} ? V_{Y}\right) / V \quad V_{Z}
$$

which X, Y, Z, and GADJ are noted as the signals input sides, and XPLX and XMNS are the signals input sides $V_{X}$ of the high-frequency carriers. Also, $V_{Y}$ represents the network signals input sides. Furthermore, $V_{Z}$ is defined as the DC voltage bias end of ZPLS and ZMNS. In addition, $V$ and $V_{W}$ are denoted as the denominator zoom input side of the WPLS and the 
WMNS. Finally, the VLC signals would be transmitted to the receiver when the modulated signals are added to the bluelight LED. Figure. 7 is our developed broadband analog multiplexing circuit.

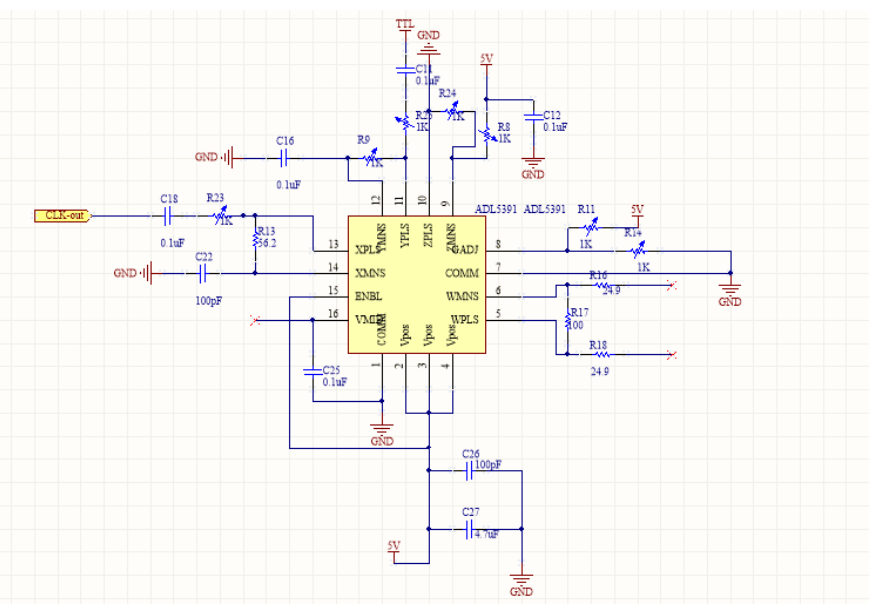

Figure 7. Broadband analog multiplexing circuit

\subsection{Mutual impedance preamplifier circuit}

Since the signals produced at the pin of the receiver are very minor with noise, it need to be enlarged with the aids of preamplifier. Here, as can be seen from Figure. 8, MAX3664 is designed as the mutual impedance preamplifier circuit and it is regarded as the filter for the analog signals. Theoretically, it is $3 \mathrm{~dB}$ bandwidth is $590 \mathrm{MHz}$.

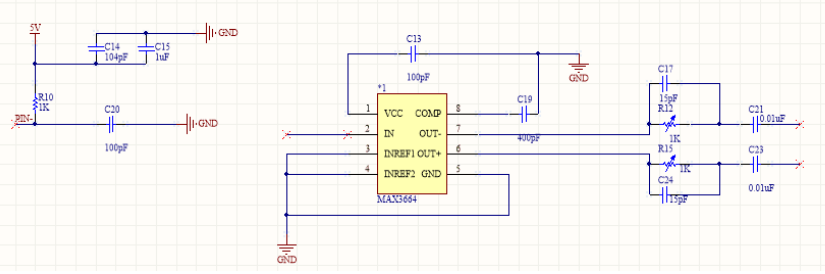

Figure 8. Mutual impedance preamplifier circuit

\subsection{TL3016 high speed comparator circuit}

We use TL3016 high speed comparator for implementing high speed comparator circuit, which is employed for transforming the signals with different types and magnitudes into rectangular signals, as shown in Figure. 9. Doing so constrains the vibration induced by shaping into the rectangular waveform. Hence, it would be a good idea to introduce the positive into the system, which can facilitate signal edge and form the hysteresis loop to remove the vibration.



Figure 9. TL3016 high speed comparator circuit

Therefore, based on the design above, we develop the miniaturization VLC system for reliable wireless transmission, which is exhibited in Figure. 10. 


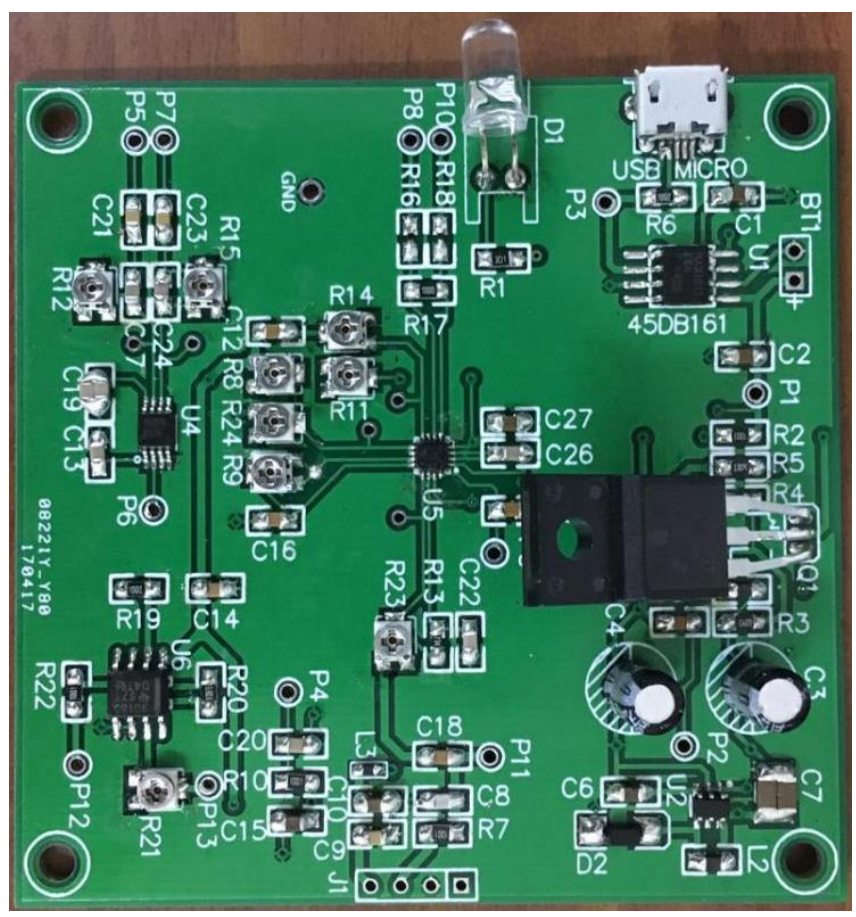

Figure 10. Chip of the developed VLC system

\section{Experiments Results and Analysis}

In this section, experiments results are presented to facilitate the performance of the newly developed VLC system. We deploy the developed VLC system and test its performance. As illustrated in Table 1, it can be observed that the proposed VLC framework achieves superior performance compared with other typical schemes.

Table 1. An example of a table
\begin{tabular}{|l|c|c|c|}
\hline Systems & Data rate (Mbps) & BER (SNR=10dB) & Distance $(\mathrm{m})$ \\
\hline Developed VLC & 100 & 0.00001 & 1.1 \\
\hline Wavelength division multiplexing VLC [1] & 450 & 0.0038 & 1 \\
\hline High-power VLC [10] & 0.266 & 0.001 & 2 \\
\hline AC sensors based VLC [14] & 8 & 0.001 & 1 \\
\hline
\end{tabular}

As depicted in Table 1, we observe that the developed VLC system outperforms other strategies in terms of the BER performance. Also, it realizes bigger data rate compared with the wavelength division multiplexing VLC system and the AC sensors based VLC system, which further demonstrates that the developed VLC system is an efficient and reliable scheme for VLC.

\section{Conclusions}

In this paper, in order to elevate the data rate and the reliability of the VLC system, we merge the FD technique with the VLC, and develop a novel FD-aided VLC system. To be specific, the proposed system can transmit the network signals directly, which can contribute to the visible light based network in the future. Also, benefited by the powerful FD, the newly developed VLC system achieve lower BER compared with previous works, and its peak rate is over $100 \mathrm{Mbps}$. Hence, we believe that the proposed VLC system is efficient and reliable for future communication scenarios.

\section{Acknowledgements}

This work was partially supported by the National Natural Science Foundation of China (61571240), and the Major Projects of the Natural Science Foundation of the Jiangsu Higher Education Institutions (16KJA510004), Jiangsu Specially Appointed Professor Grant (RK002STP16001), Innovation and Entrepreneurship of Jiangsu High-level Talent Grant (CZ0010617002), and "1311 Talent Plan" of Nanjing University of Posts and Telecommunications. 


\section{References}

1. Afgani M Z, and Haas H, Elgala H, "Visible light communication using OFDM," in Proc. 2nd International Conference on Testbeds and Research Infrastructures for the Development of Networks and Communities, 2006: 80-85.

2. L. K. Chen, and Y. Hong, "Channel-independent signal processing for high-speed VLC systems," 2017 Conference on Lasers and Electro-Optics Pacific Rim (CLEO-PR), Singapore, 2017, pp. 1-3.

3. J. Gancarz, H. Elgala, and T. D. C. Little, "Impact of lighting requirements on VLC systems," IEEE Commun. Mag., vol. 51, no. 12, pp. 34-41, Dec. 2009.

4. H. Huang, J. Wang, J. Wang, J. Yang, J. Xiong, and G. Gui, "Symbol error rate performance analysis of non- orthogonal multiple access for visible light communications," China Commun., vol. 14, no. 12, pp. 153-161, Dec. 2017.

5. H. Huang et al., "Cooperative VLC systems for data transmission and environment perception," in Proc. 2017 IEEE 9th International Conference on Communication Software and Networks (ICCSN), Guangzhou, 2017, pp. 624-629.

6. D. Karunatilaka, F. Zafar, V. Kalavally, and R. Parthiban, "LED based indoor visible light communications: State of the art," IEEE Commun. Surveys Tuts., vol. 17, no. 3, pp. 1649-1678, 3rd Quart., 2015.

7. Z. Na, Y. Wang, M. Xiong, X. Liu, and J. Xia, "Modeling and Throughput Analysis of an ADO-OFDM Based Relay-Assisted VLC System for 5G Networks," IEEE Access, vol. 6, pp. 17586-17594, Mar. 2018.

8. D. C. O'Brien, L. Zeng, H. L. Minh, G. Faulkner, J. W. Walewski, and S. Randel, "Visible light communications: challenges and possibilities," in Proc. IEEE Personal, Indoor and Mobile Radio Communications (PIMRC 2008), Cannes, France, Sep. 2008 .

9. J. Shi, X. Huang, Y. Wang, L. Tao, and N. Chi, "Improved performance of a high speed $2 \times 2$ MIMO VLC network based on EGC-STBC," in Proc. 2015 European Conference on Optical Communication (ECOC), Valencia, 2015, pp. 1-3.

10. Tsonev D, Chun $\mathrm{H}$, Rajbhandari S, et al. "A $3 \mathrm{~Gb}$ /s single-LED OFDM-based wireless VLC link using a Gallium Nitride," IEEE Photonics Technol. Lett., pp. 637-640, 2014.

11. S. Vappangi, and V. V. Mani, "Performance analysis of fast optical OFDM for VLC," in Proc. 2017 20th International Symposium on Wireless Personal Multimedia Communications (WPMC), Bali, 2017, pp. 206-211.

12. T. Yamazato et al., "Range estimation scheme for integrated I2V-VLC using a high-speed image sensor," in Proc. 2016 IEEE International Conference on Communications Workshops (ICC), Kuala Lumpur, 2016, pp. 326-330

13. H. Zhang, N. Liu, K. Long, J. Cheng, V. C. M. Leung, and L. Hanzo, "Energy Efficient Subchannel and Power Allocation for Software-defined Heterogeneous VLC and RF Networks," IEEE J. Sel. Areas Commun., vol. 36, no. 3, pp. 658-670, March 2018.

14. http://fiber.ofweek.com/2016-02/ART-210007-8420-29063911_2.html [online]. 